\title{
A Variational Principle for Eigenvalue Problems of Hamiltonian Systems
}

\author{
R. D. Benguria and M. C. Depassier \\ Facultad de Física \\ P. Universidad Católica de Chile \\ Casilla 306, Santiago 22, Chile
}

\begin{abstract}
We consider the bifurcation problem $u^{\prime \prime}+\lambda u=N(u)$ with two point boundary conditions where $N(u)$ is a general nonlinear term which may also depend on the eigenvalue $\lambda$. We give a variational characterization of the bifurcating branch $\lambda$ as a function of the amplitude of the solution. As an application we show how it can be used to obtain simple approximate closed formulae for the period of large amplitude oscillations.
\end{abstract}

2.30.Hq, $3.20+\mathrm{i}, 2.30 . \mathrm{Wd}$

Typeset using REVTEX 
Different physical systems reduce to the consideration of a one dimensional equation of the form $u^{\prime \prime}=F(u, \mu)$, where the force $F$ depends on $u$ and one or more parameters $\mu$. In these systems the energy $E=\frac{1}{2} u^{\prime 2}+V(u)$ is a constant of the motion and the qualitative dynamics can be understood from the analysis of the phase plane and the stability of the fixed points. Among the systems that fall in this class we may cite the buckling of a thin rod, the pendulum, steady state solutions of the reaction diffusion equation and numerous others [1.2.4.4, [0] (Given the large number of references in this area we refer to some of the standard textbooks only). We shall assume that the system has an equilibrium point at $u=0$. For small deviations from the equilibrium point the behavior of the solution is determined by the linear equation $u^{\prime \prime}+\lambda u=0$ with suitable boundary conditions. An important feature of the linear problem is that the eigenvalue $\lambda$ does not depend on the amplitude of the motion. For large deviations from the equilibrium, the full problem, which we write as

$$
u^{\prime \prime}+\lambda u=N(u, \lambda)
$$

must be considered. A solution to this equation and the analytic determination of the eigenvalue $\lambda$ is possible only for special forms of the nonlinearity $N$. When an analytic solution is not possible, one can resort to obtaining a numerical solution, or use perturbation theory around the linear problem. The eigenvalues of the linear part of Eq.(1) correspond to the points where bifurcation occurs in the nonlinear problem. There exist different methods of perturbation theory applicable to these systems [3,4]. An important property of the solution in the nonlinear problem is that the eigenvalue $\lambda$ depends on the amplitude of the solution.

The purpose of this article is to show that the eigenvalue derives from a variational principle. The main tool used in the derivation of this result is a new variational characterization of the linear problem which, for the sake of clarity, we consider first.

We wish to solve the linear problem

$$
u^{\prime \prime}+\lambda u=0
$$

subject to 


$$
u^{\prime}(0)=0, \quad u(1)=0
$$

where the boundary conditions we have chosen are the ones appropriate for a wide range of problems. We refer to other choices at the end. We will denote $u(0)=u_{m}$. The positive solution to this problem is given by $u_{m} \cos (\pi x / 2)$ corresponding to the lowest eigenvalue $\lambda=(\pi / 2)^{2}$. There exists a variational principle for this equation, the Rayleigh-Ritz principle which provides upper bounds on $\lambda$. This principle however cannot be extended to calculate the eigenvalue of nonlinear problems. We shall construct a new one whose extension to nonlinear problems will be straightforward.

Let $g(u)$ be an arbitrary positive function such that $g(0)=0$ and $g^{\prime}(u)>0$. Multiplying Eq.(2a) by $u^{\prime} g(u)$ and integrating in $x$ we obtain, after integrating by parts,

$$
-\frac{1}{2} \int_{0}^{1}\left(u^{\prime}\right)^{2} g^{\prime}(u) u^{\prime} d x+\lambda \int_{0}^{1} u g(u) u^{\prime} d x=0
$$

where the boundary terms vanish since $u^{\prime}(0)=0$ and $g[u(1)]=g(0)=0$. From the equation and the boundary conditions it follows that $u^{\prime}<0$ in $(0,1)$ so in the second integral we may use $u$ as the independent variable. We obtain then the identity, valid for any admisible $g$,

$$
\lambda \int_{0}^{u_{m}} u g(u) d u=-\frac{1}{2} \int_{0}^{1}\left(u^{\prime}\right)^{2} g^{\prime}(u) u^{\prime} d x
$$

Consider now, for a fixed $g$ the functional

$$
J_{g}[v]=-\frac{1}{2} \int_{0}^{1}\left(v^{\prime}\right)^{2} g^{\prime}(v) v^{\prime} d x \equiv \frac{1}{2} \int_{0}^{1} \Phi\left(v, v^{\prime}\right) d x
$$

defined for functions $v$ which satisfy

$$
v(0)=u_{m}, \quad v(1)=0, \quad \text { and } \quad v^{\prime}<0 \quad \text { in }(0,1)
$$

Then $J_{g}[v] \geq 0$ and for fixed $g$ it has a unique minimum attained at $v=\tilde{v}$. A rigorous proof will be given elsewhere. The minimizing function $\tilde{v}$ satisfies the Euler-Lagrange equation for $J_{g}$. Since $\Phi\left(v, v^{\prime}\right)$ does not depend on $x$ the Euler-Lagrange equations can be integrated once to yield $\Phi-v^{\prime} \partial \Phi / \partial v^{\prime}=$ constant. Since $\Phi\left(v, v^{\prime}\right)=-\frac{1}{2} v^{3} g^{\prime}(v)$ we get

$$
\left(\tilde{v}^{\prime}\right)^{3} g^{\prime}(\tilde{v})=-K
$$


Then, given $g$ we may determine $\tilde{v}$ by integrating Eq.(可) subject to the boundary conditions (4b). We have then, for fixed $g$,

$$
J_{g}[v] \geq \min J_{g}[v]=J_{g}[\tilde{v}]=\frac{1}{2} K\left(u_{m}\right)
$$

where the dependence of $K$ on $u_{m}$ is obtained through the boundary conditions on $\tilde{v}$.

Before going any further, consider for example $g(v)=v$. Then, the minimizing $v, \tilde{v}$, satisfies $\tilde{v}^{\prime}(x)=-K^{1 / 3}$. Imposing the boundary conditions we get $\tilde{v}=K^{1 / 3}(1-x)$ provided that $K=u_{m}^{3}$. For this choice of $g$, therefore, $J_{g} \geq u_{m}^{3} / 2$.

Now we go back to the problem under consideration. We have from equations (3), (4a) and (6),

$$
\begin{aligned}
\lambda \int_{0}^{u_{m}} u g(u) d u & =-\frac{1}{2} \int_{0}^{1}\left(u^{\prime}\right)^{2} g^{\prime}(u) u^{\prime} d x \\
& \geq J_{g}[\tilde{v}]=-\frac{1}{2} \int_{0}^{1}\left(\tilde{v}^{\prime}\right)^{2} g^{\prime}(\tilde{v}) \tilde{v}^{\prime} d x \\
& =\frac{1}{2} K\left(u_{m}\right)
\end{aligned}
$$

which gives an upper bound on $\lambda$. We have shown that for any given $g(u)$ (with $g(0)=$ $\left.0, g^{\prime}(u)>0\right)$

$$
\lambda \geq \frac{1}{2} \frac{K\left(u_{m}\right)}{\int_{0}^{u_{m}} u g(u) d u} .
$$

When will equality hold in Eq.(8)? From Eq.(7) we see that the equality will hold when $g$ is chosen in such a way that $\tilde{v}$ coincides with $u$. For this $g$ which we will call $\hat{g}$ we will have

$$
u^{\prime 3} \hat{g}^{\prime}(u)=-K
$$

Since $u$ is a solution of the differential equation (2a) we know that

$$
\frac{1}{2} u^{\prime 2}+\frac{1}{2} \lambda u^{2}=E=\frac{1}{2} \lambda u_{m}^{2}
$$

from where it follows $u^{\prime 2}=\lambda u_{m}^{2}\left(1-\left(u / u_{m}\right)^{2}\right)$. Replacing this in Eq.(9) we obtain

$$
\hat{g}^{\prime}(u)=\frac{K}{\left[\left(\lambda u_{m}^{2}\right)\left(1-u^{2} / u_{m}^{2}\right)\right]^{3 / 2}} .
$$


This equation can be integrated to yield (omitting overall multiplicative constants)

$$
\hat{g}(u)=\frac{u}{\left(1-\left(u / u_{m}\right)^{2}\right)^{1 / 2}} .
$$

Our final result for the linear problem is then the following variational characterization for the lowest eigenvalue,

$$
\lambda=\max \frac{1}{2} \frac{K\left(u_{m}\right)}{\int_{0}^{u_{m}} u g(u) d u}
$$

where the maximum is taken over all positive functions $g$ such that $g(0)=0, g^{\prime}>0$. The maximum is attained when $g=\hat{g}$.

It is straightforward to evaluate the integrals for $g=\hat{g}$ and verify that we obtain the correct result. Solving $v^{\prime 3} \hat{g}^{\prime}=-K$ with $v(0)=u_{m}, v(1)=0$ we obtain $K=(\pi / 2)^{3} u_{m}^{3}$ and $\int u g(u) d u=(1 / 2)(\pi / 2) u_{m}^{3}$ and the exact value for $\lambda$ is obtained.

We now consider the nonlinear problem

$$
u^{\prime \prime}+\lambda u=N(u)
$$

subject to

$$
u^{\prime}(0)=0, \quad u(1)=0
$$

As before we denote $u(0)=u_{m}$. Again multiplying by $u^{\prime} g(u)$, where $g$ is as before, we obtain, after integrating

$$
\lambda \int_{0}^{u_{m}} u g(u) d u=\int_{0}^{u_{m}} N(u) g(u) d u-\frac{1}{2} \int_{0}^{1}\left(u^{\prime}\right)^{2} g^{\prime}(u) u^{\prime} d x .
$$

where in the term involving $\lambda$ and the nonlinearity we have used $u$ as the independent variable. The second term in the right side is just the linear term considered above so we have

$$
\lambda \geq \frac{1}{2} \frac{\int_{0}^{u_{m}} N(u) g(u) d u+K\left(u_{m}\right)}{\int_{0}^{u_{m}} u g(u) d u}
$$

and, as before the maximum is attained when $g$ is chosen in such a way that $\tilde{v}=u$. Now the equation for $\hat{g}$ becomes, 


$$
\hat{g}^{\prime}(u)=\frac{1}{(E-V(u))^{3 / 2}}
$$

where $V(u)$ is the potential. Here the potential $V=\frac{1}{2} \lambda u^{2}-\int_{0}^{u} N(y) d y$ and $E=\frac{1}{2} \lambda u_{m}^{2}-$ $\int_{0}^{u_{m}} N(y) d y$ This expression for $\hat{g}$ cannot be integrated in general due to the nonlinear terms in $V(u)$ that arise from $N(u)$. However, as in the linear case, the maximizing $g$ exists and we obtain our main result

$$
\lambda=\max \frac{1}{2} \frac{\int_{0}^{u_{m}} N(u) g(u) d u+K\left(u_{m}\right)}{\int_{0}^{u_{m}} u g(u) d u}
$$

where the maximum is taken over all positive functions $g$ such that $g(0)=0$ and $g^{\prime}(u)>0$. The maximum is attained for $g=\hat{g}$.

Since in general we will not be able to integrate Eq.(16) the above principle gives lower bounds which can be arbitrarily close to the exact value.

As an example we shall use as a trial function the function $g$ which gives the correct eigenvalue for the linear problem $g(u)=u / \sqrt{1-\left(u / u_{m}\right)^{2}}$. With this trial function we obtain from $([17)$ the lower bound

$$
\lambda \geq\left(\frac{\pi}{2}\right)^{2}+\frac{4}{\pi u_{m}^{3}} \int_{0}^{u_{m}} \frac{N(u) u}{\sqrt{1-u^{2} / u_{m}^{2}}} d u
$$

which is valid for any $N(u)$.

As a first example consider the equation

$$
u^{\prime \prime}+\lambda u=u^{2}-\frac{7}{5} u^{3}+\frac{1}{2} u^{4}
$$

with $u(0)=u_{m}, u^{\prime}(0)=0, u(1)=0$. The integral in Eq. (18) can be done easily. We obtain

$$
\lambda \geq\left(\frac{\pi}{2}\right)^{2}+\frac{4}{\pi}\left(\frac{2}{3} u_{m}-\frac{3}{8} \cdot \frac{\pi}{2} \cdot \frac{7}{5} u_{m}^{2}+\frac{1}{2} \cdot \frac{8}{15} u_{m}^{3}\right)
$$

In Fig. 1 we show a plot of $u_{m}(\lambda)$. The solid line corresponds to the exact value obtained from the numerical integration of the equation. The dashed line corresponds to the lower bound given by the formula above. As it can be seen in the figure, the lower bound gives a very accurate value for amplitudes up to $u_{m} \approx 2$. For 
larger values there is a small discrepancy which can be minimized by a more adequate choice for $g$. The formula given in any case is a close enough lower bound even at

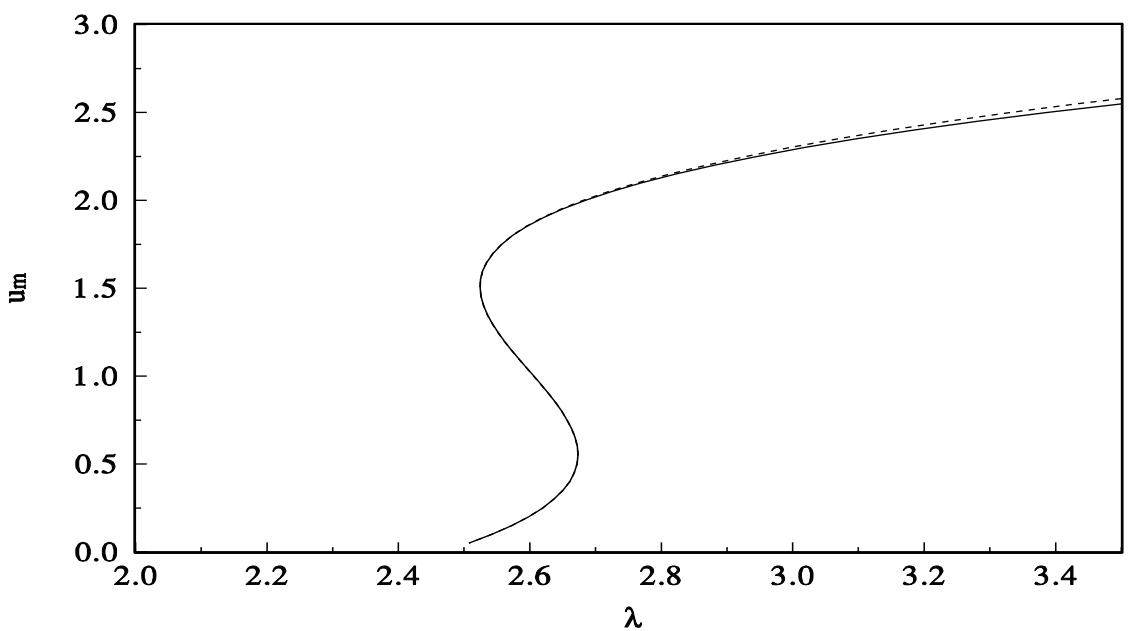

larger amplitudes.

Fig. 1

As a second example we consider the unforced Duffing equation

$$
\ddot{x}+x+\delta x^{3}=0 .
$$

For this equation $x=0$ is a stable equilibrium point and we search for the period of oscillation of perturbations from the equilibrium. This is an exactly solvable equation and the period can be given in terms of elliptic functions. When the nonlinearity is small, perturbation theory can be used to calculate the period or frequency of oscillations. The frequency obtained assuming $\delta \ll 1$ is

$$
\omega_{\text {pert }}=\frac{2 \pi}{T}=1+\frac{3}{8} \delta a^{2}-\frac{15}{256} \delta^{2} a^{4}+\text { higher order terms }
$$

where $a$ is the amplitude of the oscillations. In order to make use of the variational result we recall that since the potential has the symmetry $x \rightarrow-x$ we may consider a quarter period of the solution which we chose as the lower right quadrant in phase space $(\dot{x}, x)$. In this region of phase space we have $\dot{x}(0)=0, x(0)=a$, and $x(T / 4)=0$. Then, introducing 
the scaled time variable $\tau=2 \omega t / \pi$ we obtain

$$
\frac{d^{2} x}{d \tau^{2}}+\left(\frac{\pi}{2 \omega}\right)^{2}\left(x+\delta x^{3}\right)=0
$$

with

$$
x(0)=a, \quad \dot{x}(0)=0, \quad x(1)=0 .
$$

We identify $\lambda=(\pi / 2 \omega)^{2}, N(x)=-\delta \lambda x^{3}$ and apply Eq.(18). We obtain the bound

$$
\lambda \geq\left(\frac{\pi}{2}\right)^{2}-\frac{3}{4} \delta \lambda a^{2}
$$

which written in terms of $\omega$ gives,

$$
\omega_{\text {var }} \leq \sqrt{1+\frac{3}{4} \delta a^{2}} .
$$

This bound is valid for oscillations of any amplitude. In Fig. 2 we show the results for $\delta=0.1$. The solid line gives the exact solution, the dashed line the variational bound and the dot-dashed line the perturbation result including terms up to order $\delta^{2}$. Again we see the close agreement up to fairly large amplitude of the variational expression. As in the previous example a different trial function would give a close estimate at

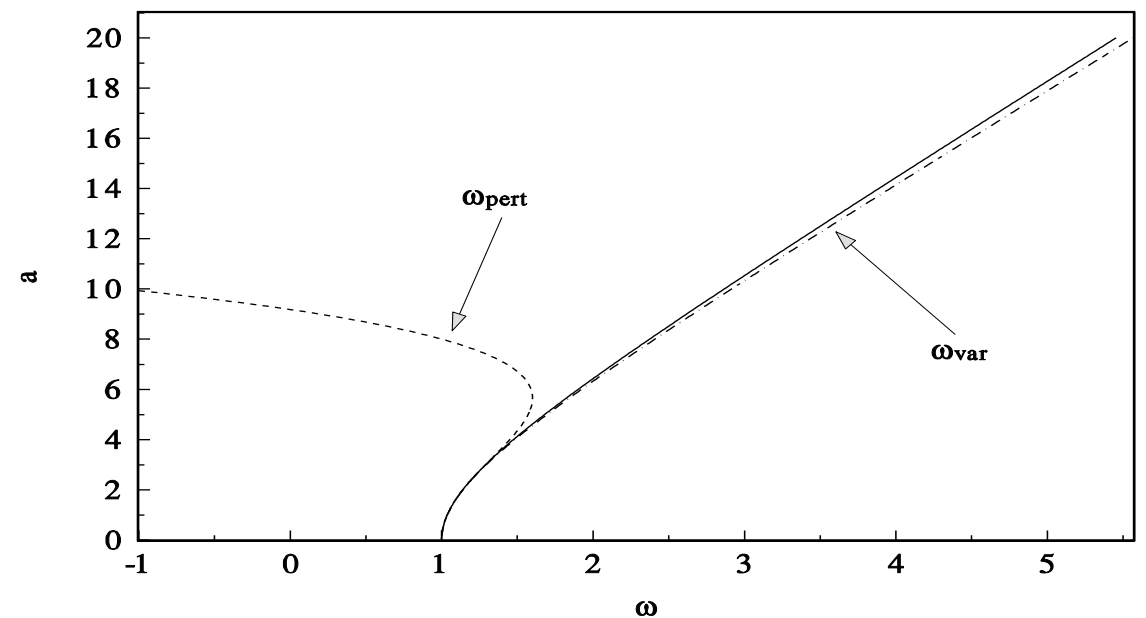

larger amplitudes.

Fig. 2 
In this last example we were able to reduce to a quarter period of the solution due to the symmetry of the potential. In the absence of such symmetry we should consider half the period, that is the lower half of the phase plane. The relevant bifurcation problem to be considered in this case is

$$
u^{\prime \prime}+\lambda u=N(u)
$$

subject to

$$
u(0)=u_{m}, \quad u^{\prime}(0)=0, \quad u^{\prime}(1)=0 .
$$

One can obtain a similar variational principle in this case too. The details are slightly more involved but entirely similar to what we have shown.

To conclude, we have shown that nonlinear eigenvalue problems for hamiltonian systems derive from a variational principle. The main tool in the derivation of this result is a new variational formulation of the linear problem. The variational principle may be used to calculate the eigenvalues as accurately as desired by suitable choice of trial functions. A simple estimate, using as a trial function the optimal trial function for the linear problem, gives reasonably accurate results for small to medium amplitude solutions, and it gives a good rigorous bound for larger amplitudes. The method that we have used here can be applied to other problems. In particular it can be shown to be a reformulation in real space of the method used previously by us [6.7] to obtain the speed of fronts of a reaction-diffusion equation in one dimension for arbitrary nonlinearities. Due to the nature of the front problem though, its formulation directly in phase space is simpler. It is an open question whether this approach can be extended to treat limit cycles of nonlinear oscillators.

This work has been partially supported by Fondecyt project 1960450. R.B. was supported by a Cátedra Presidencial. 


\section{REFERENCES}

[1] Hale, J. and Kocak H., Dynamics and Bifurcations (Springer-Verlag, New York, 1991).

[2] Keller, J. B. and Antman, S. (Eds.), Bifurcation Theory and Nonlinear Eigenvalue Problems (W. A. Benjamin, New York, 1969).

[3] Minorsky, N., Nonlinear Oscillations (Van Nostrand, Princeton, 1962).

[4] Nayfeh, A. H., Perturbation Methods (J. Wiley \& Sons, New York, 1973).

[5] Rabinowitz, P. H. (Ed.), Applications of Bifurcation Theory (Academic Press, New York, 1977).

[6] Benguria R. D. and Depassier M. C., Comm. Math. Phys. 175, 221-227 (1996).

[7] Benguria R. D. and Depassier M. C., preprint 1995. 